\title{
Editorial
}

\section{Deutsche und polnische Phraseologie und Parömiologie im Kontakt und Kontrast}

\section{Joanna Szczęk (Breslau)}

Das vorliegende Themenheft versammelt Beiträge, in denen der aktuelle Forschungsstand im Bereich der deutsch-polnischen und polnisch-deutschen Phraseologie und Parömiologie präsentiert wird. Die Idee zu diesem Themenheft ergab sich aus dem großen Interesse, dessen sich kontrastive Untersuchungen im Bereich der Phraseologie und Parömiologie erfreuen. Die Leistungen und Forschungsergebnisse der polnischen Germanistik sind daher nicht $\mathrm{zu}$ übersehen, zumal hier große Verdienste im Bereich der Phraseographie, Phraseodidaktik und der Untersuchung von semantischen Feldern in der Phraseologie aus kontrastiver Perspektive zu verzeichnen sind.

Die im Themenheft gesammelten Beiträge zeigen ein breites Spektrum im Bereich der deutschen und polnischen Phraseologie- und Parömiologieforschung. Mariusz Frąckowiak (Oppeln) analysiert phraseologische Universalien in den Phraseologismen und Sprichwörtern am Beispiel der sprachlichen Einheiten mit den Komponenten "Zeit" und "Geld". Die Studie ist kontrastiv angelegt und hat das Ziel zu präsentieren, wie beide Größen in der deutschen und polnischen Kultur wahrgenommen werden.

An die Fragen der Konzeptualisierung von bestimmten Begriffen im Deutschen geht Anna Gondek (Breslau) ein. Am Beispiel der Parömien mit der Komponente "Zorn" schlägt sie eine eingehende Analyse vor, indem sie die gesammelten Einheiten in kleinere semantische Gruppen unterteilt, die einen Beitrag zur Konzeptualisierung der Emotionen im Deutschen überhaupt leisten.

Jolanta Ignatowicz-Skowrońska und Anna Sulikowska (Stettin) liefern einen Beitrag, in dem auf die Entwicklung und den Gegenstandbereich der deutschen und der polnischen Phraseologie eingegangen wird. Die Autorinnen präsentieren einen Überblick über die Geschichte der Forschung im Bereich der deutschen und polnischen Phraseologie und unterscheiden dabei die sog. vorwissenschaftliche Phase, in der v. a. große Leistungen im Bereich der Phraseographie zu verzeichnen sind. Daran anknüpfend besprechen sie die Entwicklung der Phraseologie als wissenschaftliche Disziplin. Ergänzend werden Merkmale der sog. Phraseologizität sowie Definitionen der Phraseologismen und deren Klassifikationen thematisiert.

Eine historische Perspektive eröffnet der Beitrag von Dominika Janus (Danzig). Die Autorin analysiert die deutschen und polnischen Phraseologismen mit den Komponenten "Katze" und "Hund" in zwei Wörterbüchern: im "Słownik języka polskiego" von Samuel Bogumił Linde und im "Deutschen Wörterbuch" von Jacob und Wilhelm Grimm und ordnet sie bestimmten semantischen Feldern zu. 
Einen phraseodidaktischen Charakter hat die Studie von Marek Laskowski (Grünberg). Der Autor wählt für seine didaktischen Erwägungen phraseologische Vergleiche im Deutschen und im Polnischen, die sich sehr gut für den FSU eignen. Die Studie ist kontrastiv angelegt und im Vordergrund steht die Frage nach den Gemeinsamkeiten im Bereich der komparativen Phraseologismen in beiden Sprachen.

Text- und pragmalinguistischen Aspekten widmet Janusz Pociask (Bromberg) seine Analyse. Der Autor untersucht das Vorkommen und die Funktionen der Phraseologismen in den Pressetexten.

Semasiologisch und kontrastiv angelegt ist der Beitrag von Hanna Stypa (Bromberg). Die Autorin wählt die Phraseologismen mit tierischen Komponenten zu ihrem Untersuchungsgegenstand und geht auf die Fragen der Äquivalenz und der Divergenz ein.

Anna Sulikowska (Stettin) konzentriert sich auf die Semantik der metaphorisch motivierten Idiome und thematisiert dies aus der Perspektive der Kognitiven Grammatik. Diese Aspekte werden am Beispiel des Phraseologismus jdn. in die Zange nehmen erörtert, wobei das Ziel der Autorin ist, die semantische Vielfalt aus kognitivistischer Perspektive zu zeigen.

Jarosław Ślawski (Krakau) widmet seine Studie der Übersetzung von Phraseologismen. Als Grundlage seiner Untersuchung nimmt der Autor den Roman von Erich Kästner "Emil und die Detektive". 